\title{
Characterisation of road bumps using smartphones
}

\author{
Abhijit Mukherjee $^{1} \cdot$ Subhra Majhi ${ }^{1}$
}

Received: 25 June 2015 / Accepted: 25 April 2016 / Published online: 30 April 2016

(C) The Author(s) 2016. This article is published with open access at SpringerLink.com

\begin{abstract}
Introduction Speed bumps are used as the main means of controlling vehicle speeds all over the world. It is not too infrequent, especially in the emerging economies, to have unmarked bumps that can be perilous for the passengers. Fortuitously, the roadways and mobile phone networks have grown simultaneously in emerging economies. This paper demonstrates the capability of smartphones placed inside the vehicles in characterisation of road bumps. The smart mobile phones have accelerometers and position sensors that can be useful for autonomous monitoring roads. This can empower the user community in monitoring of roads. However, the capability of the smartphone in discerning different types of speed bumps while travelling in heterogeneous vehicle types needs to be examined.

Methods A range of road vehicles is mathematically modelled as mass, spring, and damper systems. The mathematical model of the vehicle is excited with parameters analogous to some common speed bumps and its acceleration response is calculated. The accelerometer of a smartphone is validated by comparing it with high precision accelerometers. The acceleration response of the phone while passing over the corresponding road bumps, which was used in the model earlier, is recorded using an Android based application. The experiment is repeated for different classes of vehicles. Filters have been used to reduce noise in the signals. A time averaging technique has been employed to compress the collected data.
\end{abstract}

Abhijit Mukherjee

abhijit.mukherjee@curtin.edu.au

1 Department of Civil Engineering, Curtin University, Bentley, WA 6102, Australia
Results and conclusions The acceleration signals have been digitally processed to capture road bumps. The importance of using a mathematical model to understand the acceleration response of a vehicle has been established. Also, the use of pass filters to extract the signal of concern from the noisy data has been exhibited. The ability of the technique to discern different types of speed bumps while travelling in a variety of vehicle types has been demonstrated. This investigation demonstrates the potential to automatically monitor the condition of roadways obviating costly manual inspections. As smartphones are ubiquitous, the methodology has the potential to empower the user community in the maintenance of infrastructure.

Keywords Road bumps - Smartphones · Dynamic response · Accelerometers $\cdot$ Pass filters $\cdot$ Feature recognition

\section{Introduction}

Road bumps as a speed limiting device were installed for the first time in 1906. New York Times reported it as "A Most Uncomfortable Sort of Road Devised at Chatham, N. J. [1]. Since then roads and highways are being built at an ever faster rate all over the world and the dichotomy of speed and safety continues. Despite it causing considerable discomfort and possible injuries to the passengers speed bumps have remained the main means of controlling vehicle speed and have proliferated all over the world. Moreover, an array of smaller bumps (multiple bumps) are also not uncommon. Figure 1 shows a typical single and multiple bump from an urban area.

Although there are several norms of clearly demarcating the bumps it is not too infrequent to miss the markings and they can be perilous for the passengers. Smartphones are ubiquitous and they are fitted with a number of sensors such as 
Fig. 1 Two common types of speed bumps considered in the study

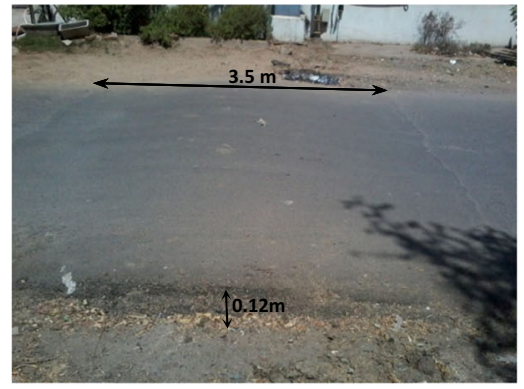

(a) Single bump

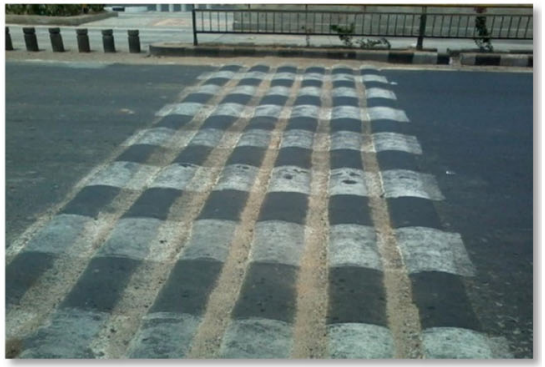

(b) Multiple bumps accelerometers and GPS. This paper explores the idea of forewarning the passengers of an approaching speed bump using a smartphone. The accelerometers of the smartphone can sense the speed bump and the GPS can determine its location. When a vehicle passes over a speed bump the smartphone can feel it and store its location. Through crowdsourcing a map of the speed bumps can be generated and it can be used by the smartphone to caution the passengers.

The methods of measuring road surface undulations has evolved over the last century [2]. Initially the surface quality measurements were based on inclinometers fitted on a bar. An operator measured the profile of the road as tilt within a specified distance by successively placing the bar. Later a wheeled system was developed as walking inclinometer [3]. Replacement of the tilt meter with an electronic dial gauge facilitated automatic data storage [4]. Moving to an acceleration based system rather than a profile based system by fitting an accelerometer and a data acquisition system on to an automobile helped in speeding up the process [5]. It also paved the way for signal processing to eliminate noise. However, monitoring the ever increasing road network through these systems is a great challenge. The emergence of smartphones and communication networks opens up new opportunities of monitoring road networks through crowdsourcing. Crowdsourcing for condition monitoring of facilities is not altogether a new concept. "Did you feel it?" is a programme through which United States Geological Survey collected information on earthquakes from the citizens who felt it [6]. Initially the information was collected in paper forms only and later, also on websites.

Along with the roads the emerging economies are rapidly building up their communication networks as well. For example India has more than a billion mobile phone connections and all the users travel on the road as well. Their phones can be the endpoints for collecting information on the roads. The road monitoring system can ride on the massive communication network to collect the road condition data from the users. Serendipitously the transport and communication networks are growing simultaneously in the regions where the challenge of road maintenance is acute. There is hope that the challenge can be alleviated by harnessing the power of the communication technology.

Among the mobile phones, only the smartphones are fitted with sensors that are capable of autogenously collecting information on road condition. Besides communication facilities, smartphones are equipped with processors, storage and sensors. Some of those sensors such as accelerometers, GPS, and magnetometers are capable of recording acceleration and its position and direction. These accelerometer records can be extremely useful in Civil Engineering, both in the laboratory and field measurements. Fortuitously, the population of smartphones is growing rapidly and they are replacing the conventional mobile phones. The prevalence of smartphones makes the task of crowd sourcing easier. There have been attempts to use smartphones crowd sourcing for mapping earthquakes [7]. They have been used for online collection of traffic data on roads $[8,9]$. This paper explores the potential of smartphones in real time monitoring road surfaces with speed bumps as a case study.

The accelerometers of smartphones have been used in speed determination of vehicles to estimate traffic congestion [9]. Research on monitoring road surface using smartphones are classified into three groups- (a) pothole detection; (b) road roughness monitoring and (c) road bump detection. An attempt to create a pothole map of Boston has been reported and the importance of eliminating spurious detections has been highlighted [10]. The signature of a smartphone located in a vehicle has been obtained and the threshold acceleration for discerning a pothole has been attempted [11]. A framework of smartphone networks connected to a central server for road monitoring has been demonstrated with potholes as an example [12]. They also discuss the importance of privacy issue in implementing the crowd sourcing network of this type. Acceleration patterns of smartphones have been explored as an inexpensive tool for road inventory identification like checking the alignment of road and determining its centre line [13]. They have also been found to be effective in detecting activities like abrupt changes in lane, and acceleration/ deceleration [14]. 
Orhan and Eren [15] report a multimodal detection tool in which the smartphone simultaneously records the images of the detected pothole by means of the tool. A road roughness coefficient has been developed and plotted on the road maps using a client server system [16]. Identification of traffic congestion by sensing frequent braking has been attempted [17]. Improvement in the accuracy of GPS signal has been attempted by applying outlier rejection and Kalman filtering to latitudes and longitudes [18]. An Android application and a website to disseminate road condition has been reported [19]. Tai et al. [20], employed support vector machine based learning to detect road anomalies and to identify their corresponding positions. Calibrating accelerations recorded by a smartphone to International Roughness Index (IRI) has also been attempted [21]. A linear relationship between the acceleration and road condition is found. A numerical study of estimate the response of road vehicle induced by surface irregularities in pavements has also been undertaken [22].

Smartphones in road condition monitoring is hitherto limited to recording of accelerations and processing them to discern potholes and monitoring overall condition of road surfaces. It is important to calibrate the phone to ascertain the veracity of the signal recorded by it. The phone is likely to be used in the heterogeneous vehicle types. Thus, it is necessary to compare the signals generated when the smartphones are used in different vehicles. In this paper, we report both a theoretical model and an experimental observation of response of a smartphone in a vehicle. In the theoretical model a spring, mass, damper system of the car is developed. Both quarter car and half car models have been formulated. The accelerations on a smooth road and a speed bump are compared. The estimated accelerations at the driver's position while the vehicle passes over a road bump are observed. The resulting acceleration-time graphs are studied to determine the dominant frequencies in the signal. In the experimental work smartphones of the inexpensive variety have been mounted on a shaker and subjected to excitation. The output of the phone is validated against the recordings of a high accuracy accelerometer of the laboratory to validate the accelerometers of the phones. The smartphones are then used in recording accelerations at the driver's position while the vehicle crosses a bump. A range of most oft used vehicles has been studied. Their response while passing over most frequently encountered bumps has been recorded. The theoretical and the experimentally observed signals have been compared.

\section{Theoretical model}

The ground vehicle can be mathematically modelled as a mass, spring, damper system [23]. The excitation due to the road surface can be applied as specified displacement at the point of contact of the wheel with the road. Application of the condition of equilibrium results in a set of linear second order differential equations of motion (Eq. 1).

$\mathbf{M U}+\mathbf{C U}+\mathbf{K U}=\mathbf{F}(\mathbf{t})$

Here ' $\mathbf{M}$ ', ' $\mathbf{C}$ ' and ' $\mathbf{K}$ ' represent the mass, damping and stiffness matrices of the system and $\mathbf{F}(\mathbf{t})$ represents the excitation force vector.

The car can be modelled with varying levels of details. The commonly used basic mathematical model to study dynamics of ground vehicle is 'Quarter Car' model where only one wheel is included. To include the interaction of two wheels 'Half Car' model is chosen with one front and one back wheel. The 'Full Car' model includes all the four wheels of the car. In this paper, we have studied the quarter car and the half car models.

\subsection{Quarter car model}

Quarter car model is a 2 degree of freedom system (Fig. 2). It has two mass points namely the sprung mass $\left(\mathrm{m}_{\mathrm{s}}\right)$ and the unsprung mass $\left(\mathrm{m}_{\mathrm{u}}\right)$. The sprung mass represents the quarter body mass of the vehicle and the unsprung mass represents the mass of one wheel of the vehicle. The spring stiffness $\mathrm{k}_{\mathrm{s}}$ and viscous damping coefficient $\left(\mathrm{c}_{\mathrm{s}}\right)$ depend on the suspension system of the vehicle. The unsprung mass is that of a single wheel. The damping of the tyre is negligible in comparison to that of the suspension. The degrees of freedom, $u_{u}$ and $u_{s}$ are the displacements of the unsprung and sprung mass respectively. In the present model it is assumed that the tyre is always in contact with the ground.

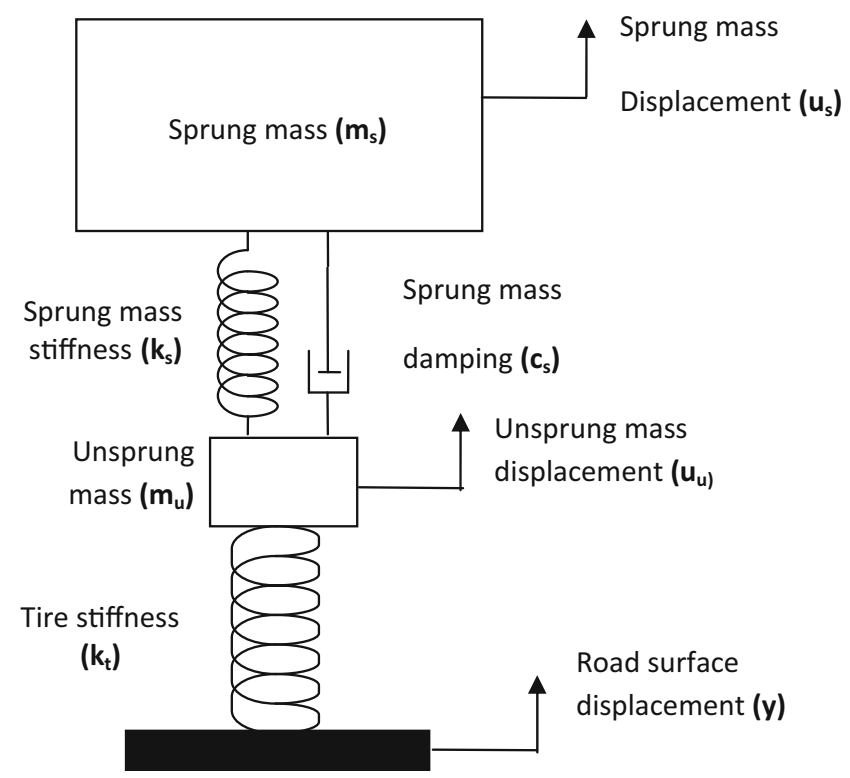

Fig. 2 The quarter car model 
Fig. 3 The half car model

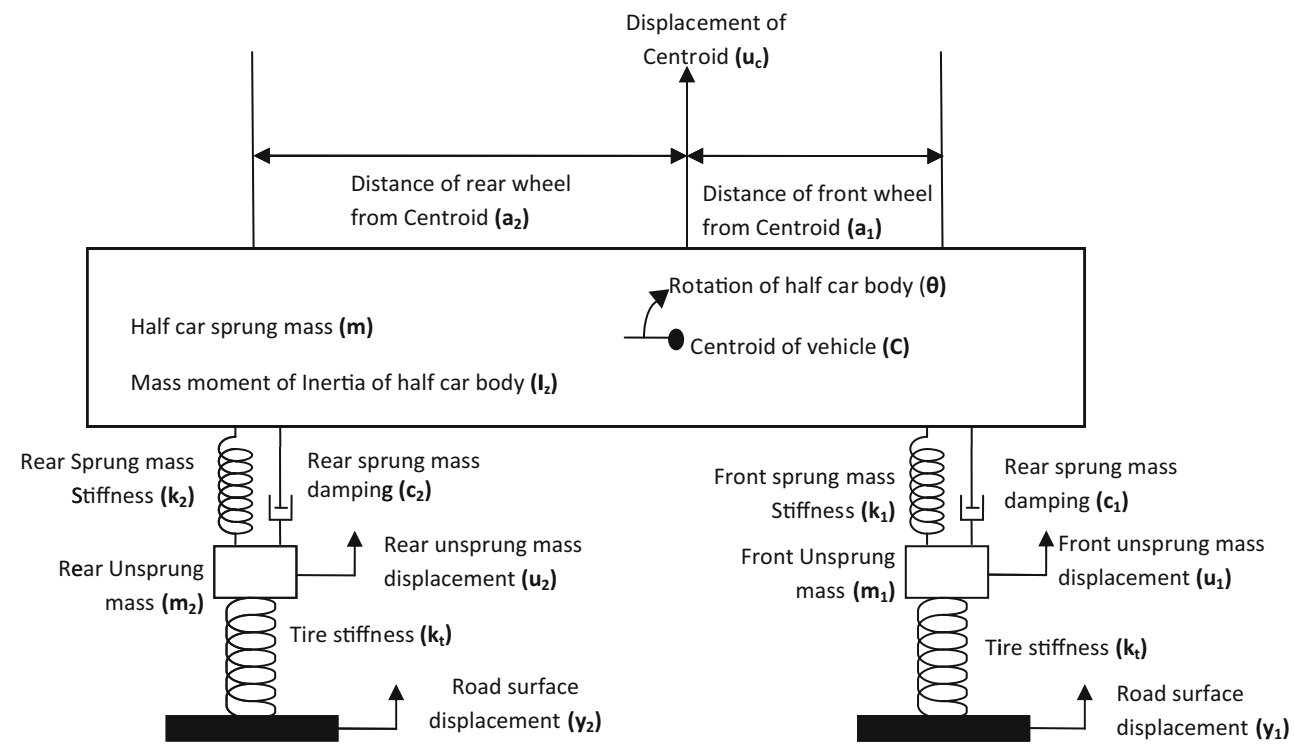

The mass, damping and stiffness matrices corresponding to the quarter car model are as follows:

$$
\begin{aligned}
& \mathbf{M}=\left[\begin{array}{cc}
m_{s} & 0 \\
0 & m_{u}
\end{array}\right] \\
& \mathbf{C}=\left[\begin{array}{cc}
c_{s} & -c_{s} \\
-c_{s} & c_{s}
\end{array}\right] \\
& \mathbf{K}=\left[\begin{array}{cc}
k_{s} & -k_{s} \\
-k_{s} & k_{s}+k_{u}
\end{array}\right]
\end{aligned}
$$

And the forcing function $\mathbf{F}(\mathbf{t})$ is given by

$$
\mathbf{F}(\mathbf{t})=\left[\begin{array}{ll}
0 & k_{u}^{*} y(t)
\end{array}\right]^{T}
$$

The displacement vector $\mathbf{U}(\mathbf{t})$ the system is given by

$$
\mathbf{U}(\mathbf{t})=\left[\begin{array}{ll}
u_{u}(t) & u_{s}(t)
\end{array}\right]^{T}
$$

The forcing function $\mathbf{F ( t )}$ in Eq. 5 can be obtained as

$$
y(t)=h^{*} \sin \left(\pi^{*} v^{*} t / l\right)
$$

Where $\mathrm{h}$ is the amplitude of the bump, $\mathrm{v}$ is the horizontal velocity of the vehicle, 1 is the width of the bump and $y$ is the displacement of the road profile normal to the surface.

\subsection{Half car model}

The quarter car model ignores the interaction of the front and the back wheels while negotiating road irregularities. When the size of the irregularity is comparable to the wheelbase it is important to consider the half car model (Fig. 3).

The wheels are modelled in the same fashion as in the quarter car model. The unsprung mass point of the front and rear section has been represented by the mass point source $m_{1}$ and $\mathrm{m}_{2}$ respectively. The spring stiffness, $\mathrm{k}_{1}, \mathrm{k}_{2}$ and viscous damping coefficient $\mathrm{c}_{1}, \mathrm{c}_{2}$ are associated with the front and rear of the vehicle. The deformation in the car body is neglected. The mass of half of the car $(\mathrm{m})$ and the mass moment of inertia $\left(\mathrm{I}_{\mathrm{z}}\right)$ is applied at the centroid $(\mathrm{C}) . \mathrm{u}_{\mathrm{c}}, \mathrm{u}_{1}$ and $\mathrm{u}_{2}$ represent the displacement of the sprung mass, front an drear unsprung masses respectively. The mass, damping and stiffness matrices corresponding to the half car model are as follows:

$\mathbf{M}=\left[\begin{array}{cccc}m & 0 & 0 & 0 \\ 0 & I_{z} & 0 & 0 \\ 0 & 0 & m_{1} & 0 \\ 0 & 0 & 0 & m_{2}\end{array}\right]$
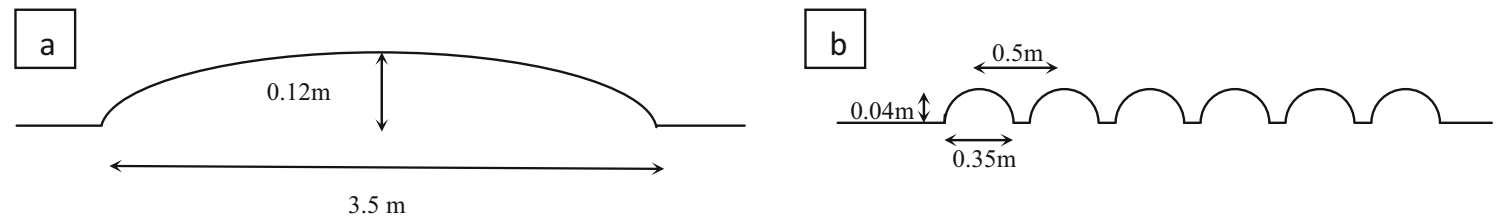

Fig. 4 Single bump and multiple with dimensions used as forcing in the model 
Table 1 Parameters used for various vehicle models

\begin{tabular}{|c|c|c|c|c|c|c|c|c|c|c|c|}
\hline Vehicle & $\mathrm{m}(\mathrm{kg})$ & $\mathrm{I}_{\mathrm{z}}\left(\mathrm{kgm}^{2}\right)$ & $\mathrm{k}_{1}(\mathrm{~N} / \mathrm{m})$ & $\mathrm{k}_{2}(\mathrm{~N} / \mathrm{m})$ & $\mathrm{m}_{1}(\mathrm{~kg})$ & $\mathrm{m}_{2}(\mathrm{~kg})$ & $a_{1}(m)$ & $\mathrm{a}_{2}(\mathrm{~m})$ & $\mathrm{c}_{1}(\mathrm{~N}-\mathrm{s} / \mathrm{m})$ & $\mathrm{c}_{2}(\mathrm{~N}-\mathrm{s} / \mathrm{m})$ & $\mathrm{k}_{\mathrm{t}}(\mathrm{N} / \mathrm{m})$ \\
\hline MUV & 800 & 1100 & 15000 & 15500 & 50 & 60 & 1.35 & 1.4 & 2000 & 2000 & 200000 \\
\hline Hatchback & 550 & 1000 & 10000 & 10500 & 40 & 50 & 1.2 & 1.3 & 1500 & 1500 & 200000 \\
\hline 2Wheeler & 200 & 500 & 13000 & 15000 & 15 & 15 & 0.8 & 1.255 & 300 & 500 & 200000 \\
\hline 3Wheeler & 150 & 500 & 13000 & 15000 & 20 & 20 & 1.2 & 0.8 & 200 & 400 & 200000 \\
\hline
\end{tabular}

$\mathbf{C}=\left[\begin{array}{cccc}c_{1}+c_{2} & a_{2} * c_{2}-a_{1} * c_{1} & -c_{1} & -c_{2} \\ a_{2} * c_{2}-a_{1} * c_{1} & a_{1}{ }^{2 *} c_{1}+a_{2}{ }^{2} c_{2} & a_{1} * c_{1} & -a_{2} * c_{2} \\ -c_{1} & a_{1} * c_{1} & c_{1} & 0 \\ -c_{2} & -a_{2} * c_{2} & 0 & c_{2}\end{array}\right]$

$\mathbf{K}=\left[\begin{array}{cccc}k_{1}+k_{2} & a_{2} * k_{2}-a_{1} * k_{1} & -k_{1} & -k_{2} \\ a_{2} k_{2}-a^{*} k_{1} & a_{1}{ }^{2 *} k_{1}+a_{2}{ }^{2 *} k_{2} & a_{1} * k_{1} & -a_{2} * k_{2} \\ -k_{1} & a_{1} * k_{1} & k_{1}+k_{t} & 0 \\ -k_{2} & -a_{2} * k_{2} & 0 & k_{2}+k_{t}\end{array}\right]$

The force vector for this system represented by $\mathbf{F ( t )}$ is

$\mathbf{F}(\mathbf{t})=\left[\begin{array}{llll}0 & 0 & k_{t}^{*} y_{1}(t) & k_{t}^{*} y_{2}(t)\end{array}\right]^{T}$

The displacement vector $\mathbf{U}(\mathbf{t})$ the system is given by

$\mathbf{U}=\left[\begin{array}{llll}u_{c}(t) & \theta(t) & u_{1}(t) & u_{2}(t)\end{array}\right]^{T}$

Similarly, in case of a half car model, the displacement term in the forcing function for the front wheel is formulated as per Eq. 10. The forcing function for rear wheel is exactly the same for the front wheel, but is incident after a time lag equal to the time required for the rear wheel to reach the bump after the crossing of the front wheel over the bump.

The prime difference between this model and the quarter car model in the present context is that the effect of variation of wheelbase of the vehicle can be accounted for in the half car model. Thus different classes of vehicles can be modelled and studied through this model. We shall compare the responses of the quarter car and the half car models later in the paper.

Equation 1 is solved through direct integration in time. Average acceleration assumption of Newmark's Beta method is used to solve the discretised differential equation [24].

$\mathbf{M} \ddot{\mathbf{U}}_{i+1}+\mathbf{C} \mathbf{U}_{i+1}+\mathbf{K} \mathbf{U}_{i+1}=\mathbf{F}(\mathbf{t})_{i+1}$

The time velocity and displacement at the next time step are determined as:

$\mathbf{U}_{i+1}=\mathbf{U}_{i}+\left[0.5^{*} \Delta t\right] \ddot{\mathbf{U}}_{i}+\left[0.5^{*} \Delta t\right] \ddot{\mathbf{U}}_{i+1}$

$$
\begin{aligned}
\mathbf{U}_{i+1}= & \mathbf{U}_{i}+(\Delta t) \mathbf{U}_{i}+\left[0.25^{*}(\Delta t)^{2}\right] \ddot{\mathbf{U}}_{i} \\
& +\left[0.25^{*}(\Delta t)^{2}\right] \ddot{\mathbf{U}}_{i+1}
\end{aligned}
$$

In this case, values of beta and gamma are 0.25 and 0.5 respectively.

The width and height at the apex of the bump for the single bump considered for the single bump was $3.5 \mathrm{~m}$ of $0.12 \mathrm{~m}$. And, in case of multiple bumps, the width and height at apex for each bump was $0.35 \mathrm{~m}$ and $0.04 \mathrm{~m}$ respectively as shown in Fig. 4. The parameters used in the model of various vehicles is presented in Table 1 .

The masses of the MUV, hatchback and 3 wheeler vehicle listed in Table 2 are their corresponding 'half car' masses, i.e., half of their total masses. While one quarter of their masses was used in the quarter car model.

\section{Experimental investigation}

The main aim of this investigation is to examine if smartphones can be used in monitoring roadway surfaces. Thus, their ability to reliably record acceleration is paramount. As a large variety of smartphones exist it is important to understand their variations in terms of accelerometers fitted in them. We choose a basic model of Android ${ }^{\circledR}$ smartphone, costing around $\$ 70$ that is likely to have simple accelerometers of lowest precision. The accelerometers are queried through an application written to acquire acceleration in the three axes at a specified sampling rate. The sampling rate was set at $100 \mathrm{~Hz}$.

Table 2 Nomenclature of the vehicles and bumps

\begin{tabular}{lll}
\hline Vehicle type & \multicolumn{2}{l}{ Bump type } \\
\cline { 2 - 3 } & Single bump & Multiple bumps \\
\hline 4-Wheeler MUV & 4 WMSB & 4 WMMB \\
4-Wheeler Hatchback & 4 WHSB & 4 WHMB \\
2-Wheeler & $2 \mathrm{WSB}$ & $2 \mathrm{WMB}$ \\
3-Wheeler & $3 \mathrm{WSB}$ & $3 \mathrm{WMB}$ \\
\hline
\end{tabular}




\subsection{Validation of the accelerometers}

The accelerometers of the smartphone are tested in the laboratory by comparing their records with that of high precision laboratory accelerometers. The smartphone has been attached to a uniaxial shake table (Fig. 5).

Accelerometers were attached to the phone. They were connected to signal conditioners and a data acquisition system (National Instruments). The response of the smartphone was recorded directly on its own storage device. One notices the striking miniaturization of the data acquisition system in case of the smartphone. In case of the laboratory system one needs the accelerometers, the signal conditioner, the data acquisition system and the computer that can barely move out of the laboratory. In case of the smartphone all these are integrated into one device that can fit one's palm. Thus, the smartphone is extremely convenient for both field and laboratory use. The cost of the smartphone is also hugely lower than that of the laboratory system.

It was noticed that there was very good correlation between the record of the smartphone and the accelerometer in the entire range of excitation $(3-20 \mathrm{~Hz})$. The two records $(8 \mathrm{~Hz}$ and $3 \mathrm{~Hz}$ ) that showed a little deviation are presented in Fig. 6.

A critical analysis of the performance of smartphones in recording vibration is beyond the scope of this paper. The main aim of this investigation is to assess the suitability of smartphones in monitoring road surfaces. It is concluded from this experiment that the smartphones are suitable for such tasks.

\subsection{Recording accelerations at road bumps}

To record the accelerations of vehicles a set of speed bumps located on the main roads of Ahmedabad, India was identified. We measured the dimension of the bump, i.e. the width and height of the bump. The smartphone was secured at the

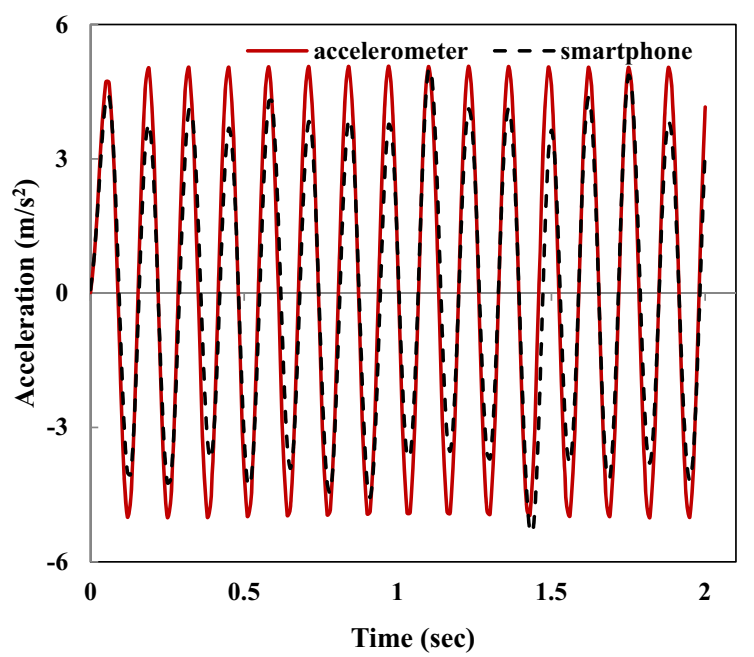

(a) $8 \mathrm{~Hz}$

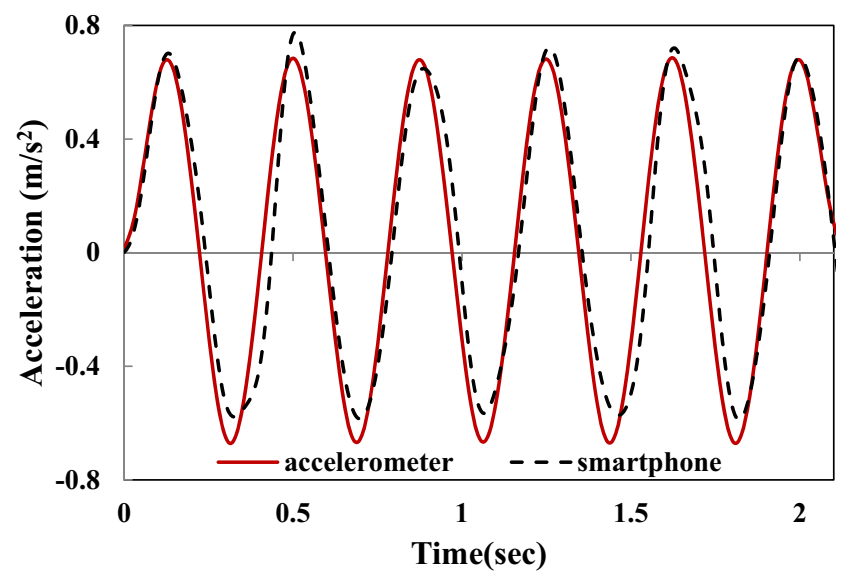

(b) $3 \mathrm{~Hz}$

Fig. 6 Accelerations recorded by the accelerometer and the smartphone

centre of the dashboard. Using the Android app specially written for this purpose the accelerations in three axes were

Fig. 5 The experimental setup

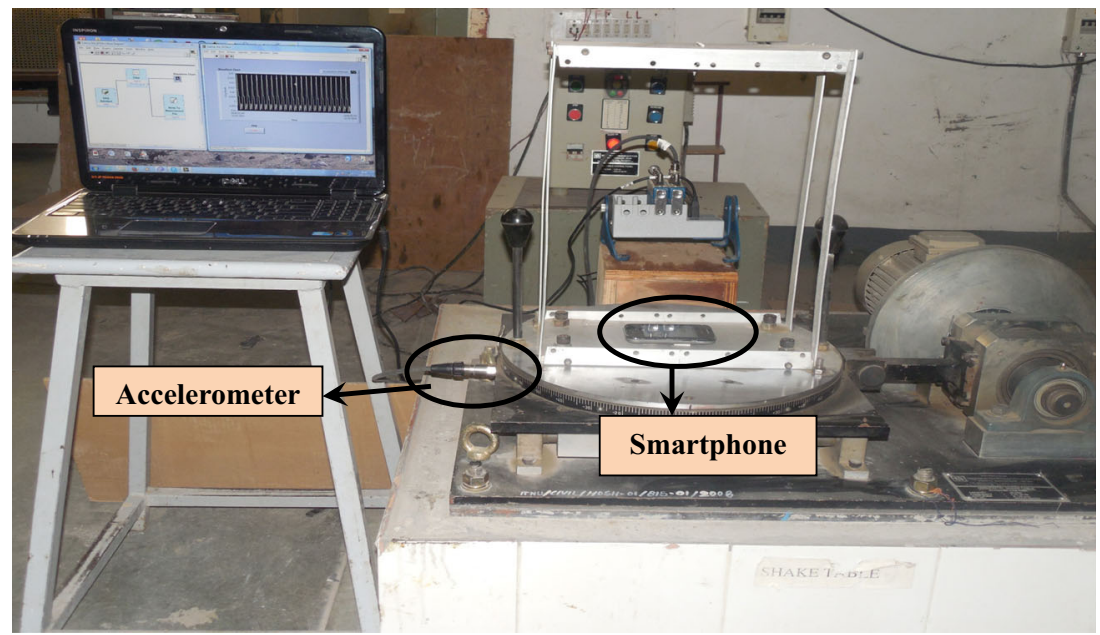


recorded while crossing the speed bump. Two most common types of bumps are considered in these examples. In the first scenario, a single bump of width $3.5 \mathrm{~m}$ and height $0.12 \mathrm{~m}$ is considered (Fig. 1a).

Next, multiple bumps, which comprise of a series of 6 bumps, of width $0.35 \mathrm{~m}$ and height $0.04 \mathrm{~m}$ each and separated by of $0.5 \mathrm{~m}$ from centre to centre is considered (Fig. 1b). The acceleration recorded by the smartphone is processed further.

\subsection{Data processing}

The acquired data consists of accelerations due to all motions. To highlight the accelerations due to road surface the records have been filtered. The filter is applied in the frequency domain of the acceleration signal. For this purpose, the data acquired in the time domain is converted into frequency domain by using Discrete Fourier Transform (DFT) . The frequency domain data are filtered to retain the frequencies of interest using a low pass filter. The filtered data are converted back from frequency domain to time domain.

\subsection{Post processing}

To extract the pattern of acceleration while passing over a bump the filtered accelerations are compressed by averaging their absolute values over each second:

$\bar{x}_{p}=\sum_{i=1}^{p_{j}}\left|x_{i}\right| / p_{j}$

Where $p_{j}=$ number of sampling points in jth second

The average $(\bar{x})$ is in turn scaled by the maximum value:

$\overline{\bar{x}}=\left.\bar{x}_{p}\right|_{\bar{x}_{\max }}$

The results are discussed in the next section.

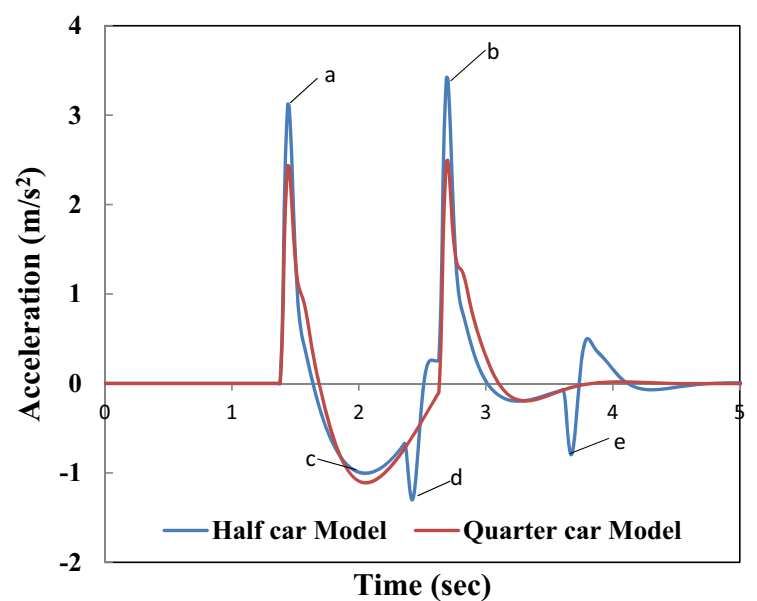

(a) Single bump

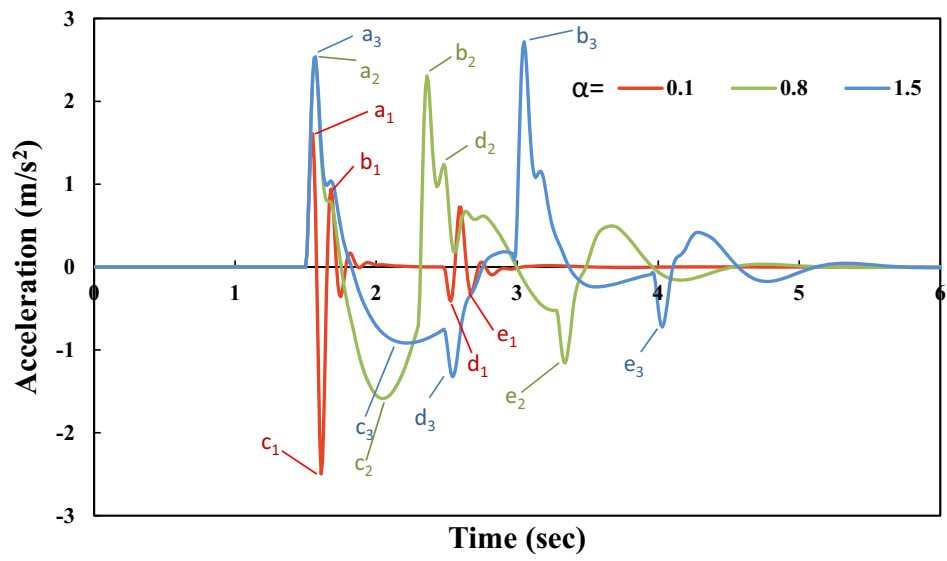

(b) Varying $\alpha$

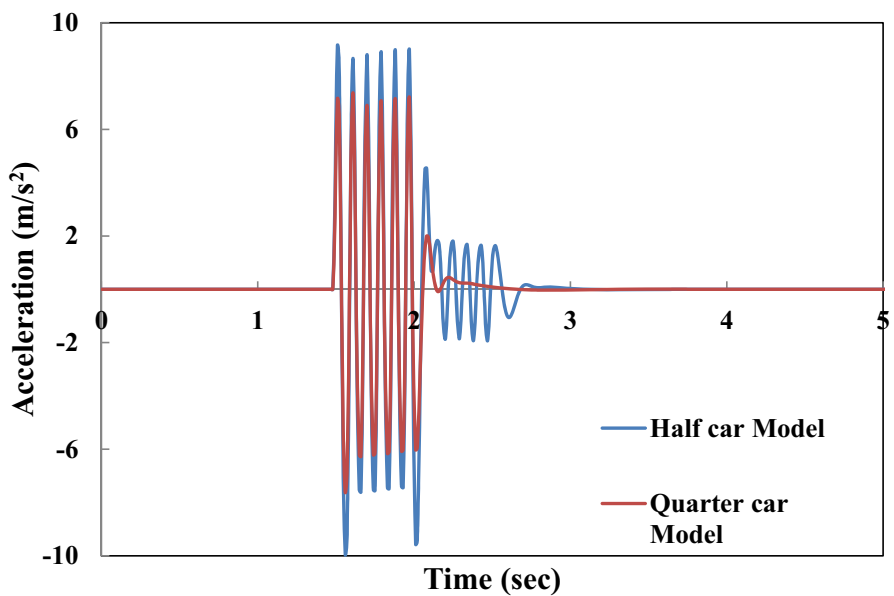

(c) Multiple Bumps

Fig. 7 Computed acceleration responses over speed bumps 


\section{Results and discussion}

This section discusses the experimental and theoretical results. Various theoretical models have been compared. Response of vehicles on different bumps has been presented. Effect of data

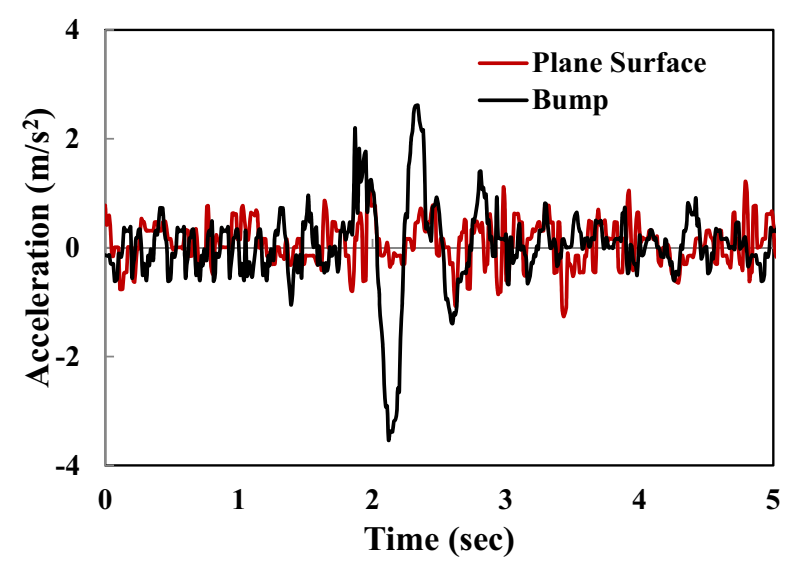

(a) Data as measured

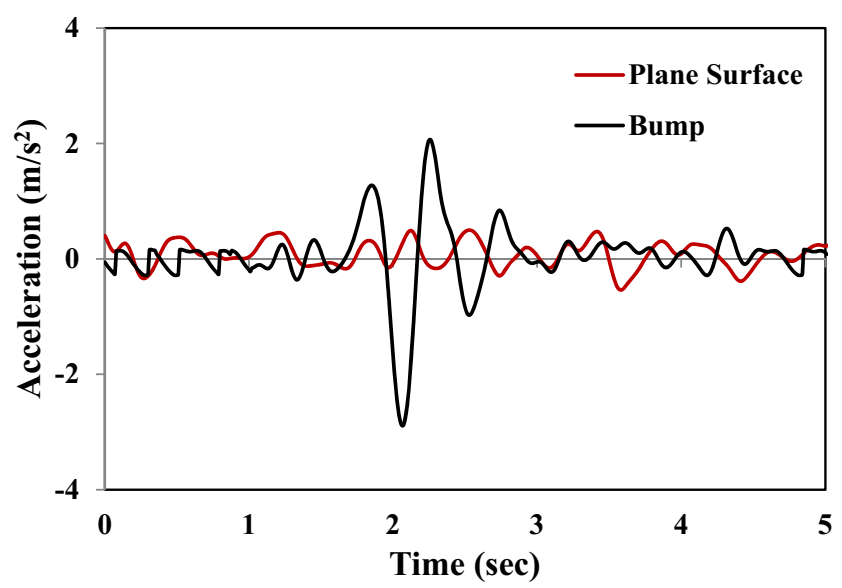

(b) Filtered Data

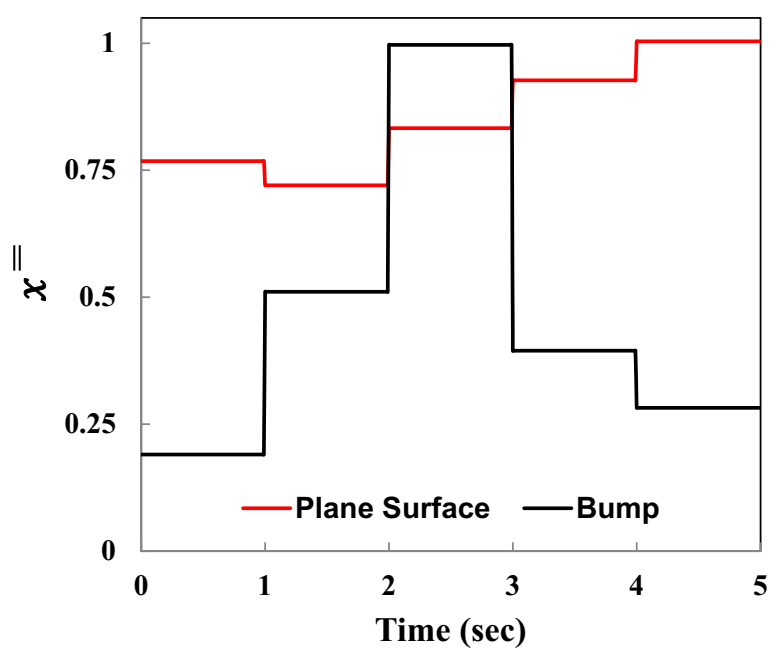

(c) Compressed data

Fig. 8 Acceleration recorded on plane road and bumps in an MUV processing has been demonstrated. The time data has been compressed to discern the bumps from the level road. Finally, the response of various classes of vehicles has been compared.

\subsection{Quarter car and the half car model}

Figure 7 presents the accelerations computed by the half car and the quarter car models for the bumps. It may be noted in case of the quarter car model the responses are recorded on the sprung mass position. In case of the half car model the records are at the driver's position. The half car model also includes the interaction between the front and the rear wheels, which is absent in the quarter car model. The quarter car model closely follows the response of the half car model at all these peaks. The positive peaks $(a, b)$ of the half car model are higher than those of the quarter car model. In case of the half car model additional smaller peaks $(\mathrm{d}, \mathrm{e})$ appear that are not predicted in the quarter car model. Understandably, these peaks are due to the rear wheel. Clearly, the effect of the rear wheel is not as pronounced as in case of the front wheel.

In case of single bump (Fig. 7a) first positive peak ' $a$ ' appears is at the instant when the front wheel starts to ride the bump. The second positive peak ' $b$ ' appears when the front wheel leaves the bump. A gentler negative peak ' $c$ ' appears in between the two sharp positive peaks when the front wheel has reached the crown of the bump and starts to descend it. To examine this phenomenon we present the response for three bump width to vehicle wheel base ratios $(\alpha)$. When the bump width is larger than the wheel base the front wheel $(\alpha=$ 1.5) the rear wheel starts to climb the bump before the front wheel has left it. Thus, peak $d$ appears in between the peaks ' $a$ ' and ' $b$ '. In case of $\alpha=0.8$ the relative positions of the peaks get changed. The peaks, ' $d$ ' and 'e' appear after peaks ' $a$ ' and 'b', because the rear wheel starts to climb the bump only after the front wheel has left it. We observed the overlap between the peaks ' $b$ ' and ' $c$ ' when $\alpha$ is close to 1 . In the case of extremely narrow bumps $(\alpha=0.1)$ the peaks due to the front

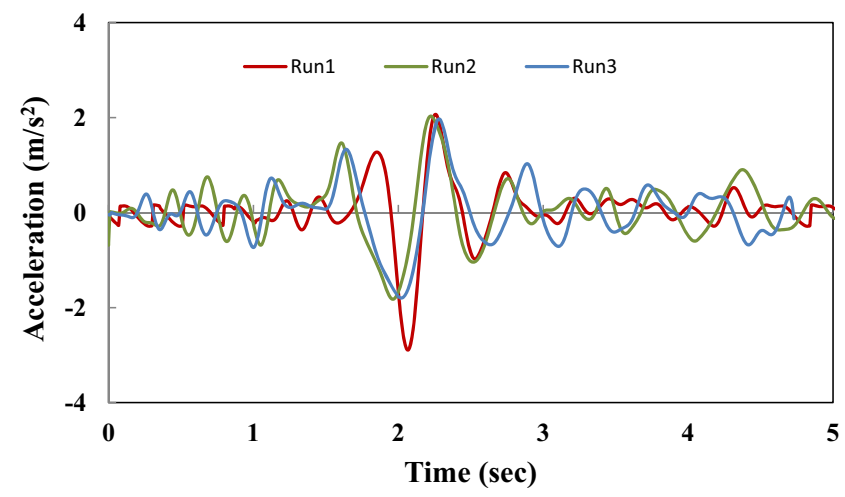

Fig. 9 Acceleration recorded in an MUV with different drivers 
wheel ('a', 'b' and 'c') are well separated from the peaks due to the rear wheels ('d' and ' $e$ ').

In case of multiple bumps (Fig. 7c) the large peaks due to the front wheel are predicted by both the half car and the quarter car models. The following smaller peaks due to the rear wheel riding over the bumps are predicted by the half car model only. Hence, it can be concluded that the peaks due to

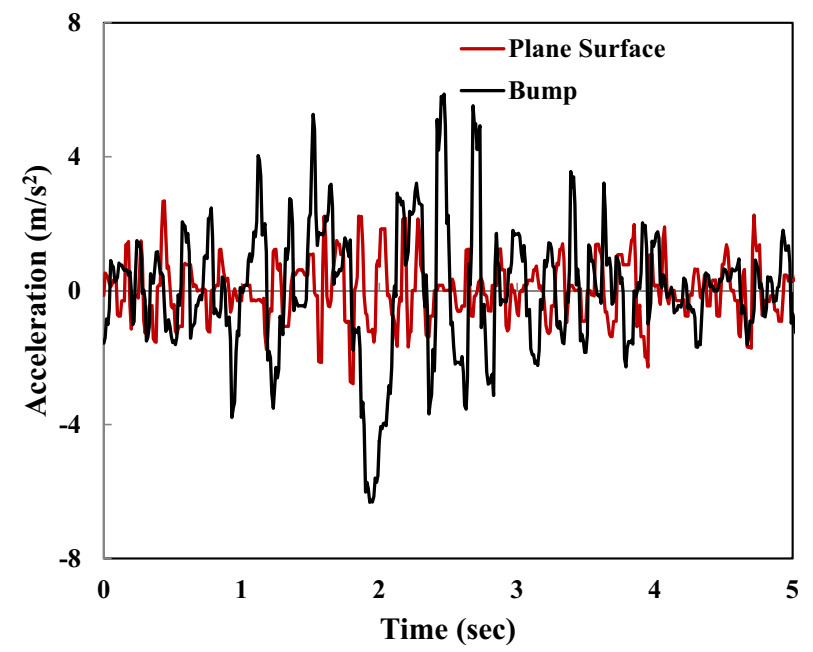

(a) Data as measured

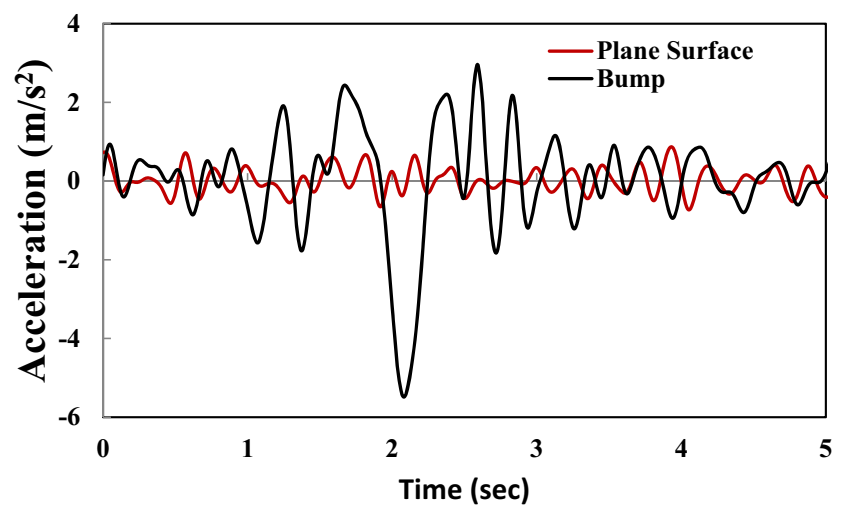

(b) Filtered Data

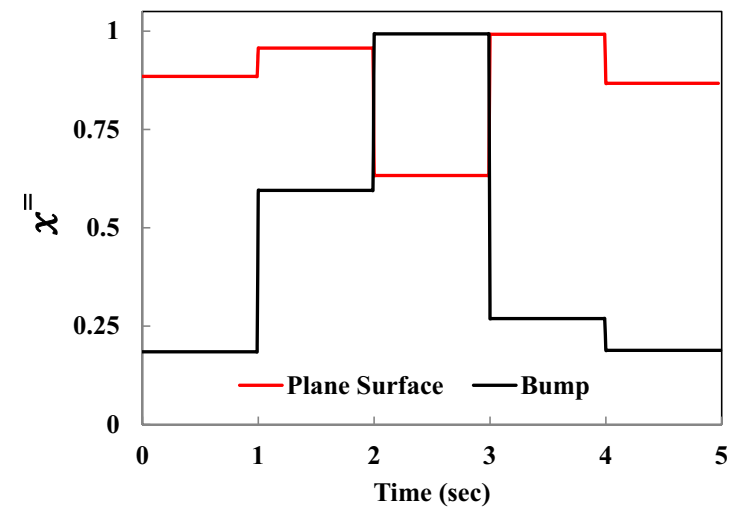

(c) Compressed data

Fig. 10 Accelerations recorded in a 3-wheel vehicle

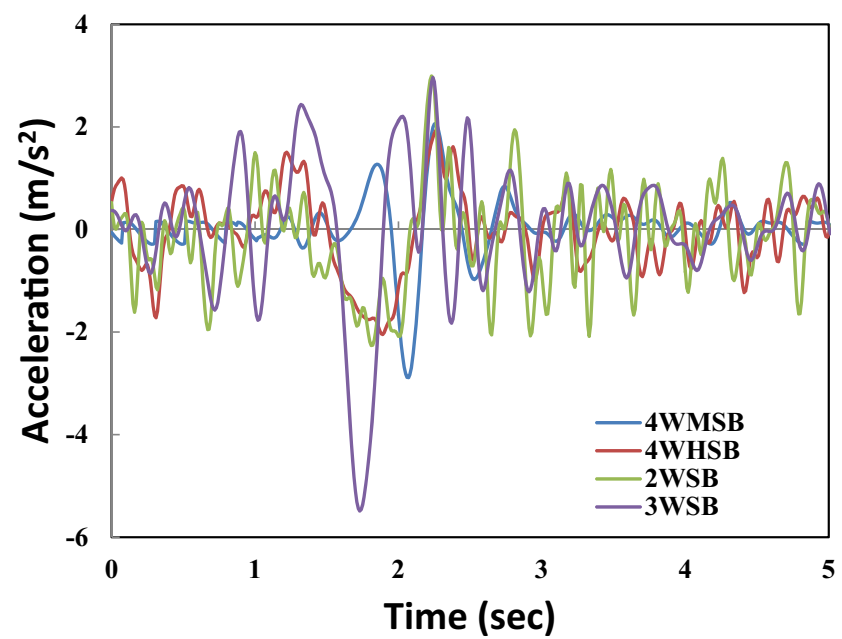

Fig. 11 Filtered acceleration for different classes of vehicles

the rear wheel are smaller and may be insignificant. In this case, as the wheels come down from a bump they immediately start to climb the following bump. Thus, peak ' $b$ ' of the preceding bump merges with the peak ' $a$ ' of the following bump. The models have been compared with the experimentally observed accelerations later in the paper.

\subsection{Experimental results}

The experimental data has been collected using a smartphone at the driver's position of a multi-utility vehicle (MUV). The data have been processed as described earlier. Figure 8 a shows the records of the smartphone on plane roads and bumps. It may be noted that the average accelerations in both cases is around $10 \mathrm{~m} / \mathrm{s}^{2}$. This is because the accelerometer of the

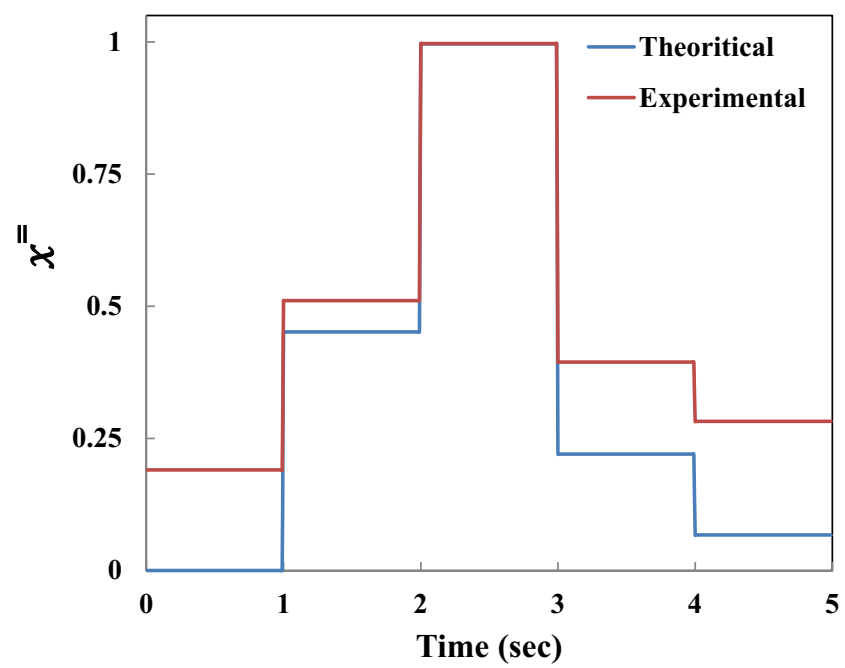

Fig. 12 Compressed accelerations corresponding to 4WMSB 
Table 3 Response ( $x^{=}$) of different vehicles crossing single bump

\begin{tabular}{|c|c|c|c|c|c|c|c|c|}
\hline \multirow[t]{3}{*}{$\left(x^{=}\right)$} & \multicolumn{8}{|c|}{ Vehicle type } \\
\hline & \multicolumn{2}{|l|}{ 4WMSB } & \multicolumn{2}{|l|}{ 4WHSB } & \multicolumn{2}{|l|}{$2 \mathrm{WSB}$} & \multicolumn{2}{|l|}{$3 \mathrm{WSB}$} \\
\hline & Theoretical & Experimental & Theoretical & Experimental & Theoretical & Experimental & Theoretical & Experimental \\
\hline 1 & 0 & 0.192 & 0 & 0.182 & 0 & 0.349 & 0 & 0.078 \\
\hline 2 & 0.451 & 0.519 & 0.448 & 0.445 & 0.252 & 0.446 & 0 & 0.126 \\
\hline 3 & 1 & 1 & 1 & 1 & 1 & 1 & 1 & 1 \\
\hline 4 & 0.223 & 0.394 & 0.153 & 0.239 & 0.210 & 0.394 & 0.508 & 0.269 \\
\hline 5 & 0.06 & 0.282 & 0.017 & 0.293 & 0.022 & 0.397 & 0.022 & 0.070 \\
\hline
\end{tabular}

smartphone includes acceleration due to gravity. Thus, the average acceleration in both bump and level surface is the acceleration due to gravity.

As the vehicle crosses the bump the accelerations are clearly higher than that on level road. The signature of the bump noticed in the theoretical model is clearly visible in the measured acceleration. Thus, it can be concluded that smart phones are capable of discerning the bumps on roads. However, there are high frequency components in the records that are not observed in the theoretical model. The noise might have been caused by the transmitted vibration from the engine and drive systems. The high frequency components have been filtered from the data by means of a low pass filter set at $4 \mathrm{~Hz}$ (Fig. 8b).

Clearly, removal of the high frequency components enhances the contrast between the bump and the level road. The filtered accelerations follow closely the theoretically predicted response. The filtered acceleration has been further processed as in Eqs. 16 and 17 to create the signature of the bump (Fig. 8c). The value of acceleration due to gravity (g) has been subtracted from the acceleration values before processing the data.

In case of the compressed data the bump is discerned through just five average acceleration records. Thus, it is possible to keep the record of the bump through just five floating point numbers. The irregularities in the roads are likely to be numerous. A compressed representation of the bump allows recording them in a smartphone with frugal storage and transmitting them to the central server with very modest data size. It should allow recording the irregularities of a large road network in a very economical manner and storing the information efficiently.

It is important to test the repeatability of the records by the smartphone. The accelerations were recorded repeatedly while crossing the bump in the same vehicle driven by different drivers (Fig. 9).

The signature of the bump is clearly observed in all the records. The signatures are very close to one another. Thus, records of the smartphone are reliable. The MUV used in the previous example is a standard vehicle used worldwide. The present investigation is focussed to the emerging economies where a heterogeneous population of road vehicles exists. A unique three wheel vehicle is very popular as a prime mover on those roads. Dimensions, mass and suspension system of these vehicles is very different from that of the MUV. It is important to examine the performance of the present system for such vehicles. Figure 10 presents the raw data, filtered data and compressed data for the threewheeler. It is clear from Fig. 10a that the noise recorded in these vehicles is higher than that of the MUV. The suspension system of these vehicles transmits higher noise to the driver's position. It, however, has been filtered effectively (Fig. 10b) and the signature of the bump is clearly discernible. When the data are compressed the contrast between the bump and the level road is as clear as in case of MUVs. Thus, the present system works satisfactorily in case of three wheelers as well.

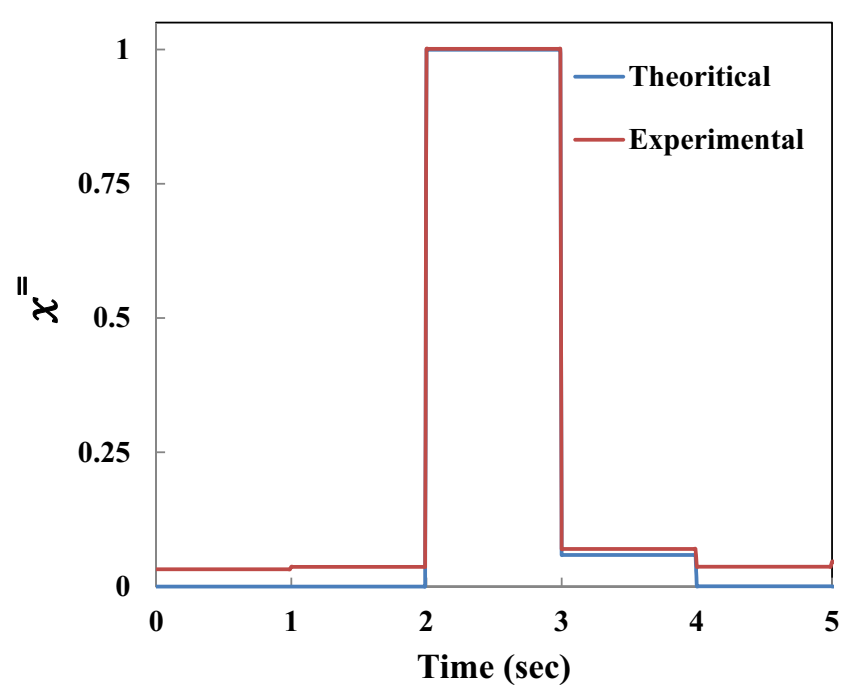

Fig. 13 Compressed acceleration corresponding to $4 \mathrm{WHMB}$ 
Table 4 Response ( $x^{=}$) of different vehicles crossing multiple bumps

\begin{tabular}{|c|c|c|c|c|c|c|c|c|}
\hline \multirow[t]{3}{*}{$\left(x^{=}\right)$} & \multicolumn{8}{|l|}{ Vehicle type } \\
\hline & \multicolumn{2}{|l|}{ 4WMMB } & \multicolumn{2}{|l|}{ 4WHMB } & \multicolumn{2}{|l|}{ 2WMB } & \multicolumn{2}{|l|}{$3 \mathrm{WMB}$} \\
\hline & Theoretical & Experimental & Theoretical & Experimental & Theoretical & Experimental & Theoretical & Experimental \\
\hline 1 & 0 & 0.126 & 0 & 0.032 & 0 & 0.0904 & 0 & 0.078 \\
\hline 2 & 0 & 0.109 & 0 & 0.036 & 0 & 0.18 & 0 & 0.126 \\
\hline 3 & 1 & 1 & 1 & 1 & 1 & 1 & 1 & 1 \\
\hline 4 & 0.257 & 0.688 & 0.069 & 0.069 & 0.357 & 0.262 & 0.508 & 0.269 \\
\hline 5 & 0.002 & 0.237 & 0 & 0.036 & 0 & 0.052 & 0.022 & 0.070 \\
\hline
\end{tabular}

\subsection{Parametric study}

With the success of the present method in two widely varying classes of road vehicles a parametric study is carried out to evaluate the applicability of the present method for all classes of vehicles. These vehicles vary widely in their masses, dimensions, wheels and suspension systems. This section explores the applicability of the technique in all classes of vehicles. Table 2 presents the classes of vehicles included. Most popular vehicles on Indian roads have been included here. Both single and multiple bumps have been included. For the case of single bump it was observed that vehicles of all classes, except 2WSB, the vehicles crossed the bump at $10 \pm 2$ $\mathrm{Km} / \mathrm{h}$. In case of $2 \mathrm{WSB}$ the velocity was $15 \pm 2 \mathrm{Km} / \mathrm{h}$. For the vehicles crossing the multiple bumps, the speed was found to be $20 \pm 3 \mathrm{Km} / \mathrm{h}$ for all classes of vehicles.

The filtered accelerations for the vehicles are presented in Fig. 11. It can be seen that although the bump is visible in all the records they vary significantly among different classes of vehicles. The two-wheeler, another extremely popular vehicle, has significantly larger number of cyclic oscillations than the rest. It may be due to the different class of suspensions in these vehicles and very different position of the rider. The threewheeler has significantly higher transmission of oscillation through the suspension. The hatch back and the MUV also differ in their response to bumps. It is important to compare the compressed data for all these vehicles with the theoretically computed responses.

Figure 12 presents the compressed response for $4 \mathrm{WMSB}$ crossing a bump. There is a great deal of similarity among the records. Clearly, the experimental measurement and theoretical predictions agree very well. Thus, it is clear that smartphones can record road bumps very accurately while riding all classes of vehicles. The present theoretical model is able to model the vehicles of all popular classes. Across all the vehicle classes a clear peak is observed and its temporal location remains unchanged. The second largest ordinate is either to the right or the left of the largest peak. The second largest ordinate is nearly $50 \%$ lower than the peak in all cases. The first and the last ordinates are the lowest in all cases. Thus, it can be concluded that regardless of the type of vehicle the compressed signature is very similar.

Compressed response $\left(x^{=}\right)$values of different vehicles crossing the single bump viz., 4WMSB, 4WHSB, 2WSB and 3 WSBhave been presented in Table 3.

Figure 13 presents the response of all classes of vehicles while crossing multiple bumps. The agreement between the theoretical predictions and the experimental records is very close. The peak was very clear in this case as well and it remained at the same position as in case of the single bump. The second largest ordinate was to the right of the peak. The difference between the peak and the second largest ordinate was even more dramatic in this case. The lowest ordinates were at the beginning and the end of the records. Thus, the present system captures the multiple bumps very clearly. Other cases of vehicles crossing the single viz., 4WHSB, $2 \mathrm{WSB}$ and $3 \mathrm{WSBhave}$ been compared with case 4WMSB in Table 4.

\section{Concluding remarks}

The present paper describes a method of detection of road irregularities using the accelerations recorded by the smartphones of drivers of different classes of vehicles. The vehicles included the MUVs, hatchbacks, 3-wheelers and 2wheelers that constitute all the major classes of vehicles in a diverse population of automobiles that travel the roads of an emerging economy such as India. The ability of the smartphones in recording accelerations reliably is demonstrated. A theoretical model of the vehicles is described using both quarter car and half car models. The signatures of both single and multiple bumps are developed. The records of the smartphones are filtered and compressed to highlight the effect of the bumps. It is noted that although the signature of the bump can be clearly seen in all the signals they vary considerably with different classes of vehicles. The ability of the 
filtration and compression techniques in highlighting the road irregularities is demonstrated.

As the emerging economies build their road network they face a formidable challenge of monitoring these roads. It seems unlikely that the conventional monitoring techniques that depend on specialised vehicles with skilled technicians, will be able to cope with this challenge. As the number of vehicles and the size of the road network develop so does the number of smartphones and the size of the communication network. The present technique leverages the growth of smartphones and communication networks to monitor the roads automatically. Through crowd sourcing techniques the users of the road networks can be made responsible for their maintenance. However, several social and privacy issues must be addressed to deploy the technology. Also the scaling ability of the technology for a large network needs to be demonstrated. The present paper validates that at the client end the technology is very reliable and efficient. Experimentation is underway to test its scalability.

Acknowledgments The authors acknowledge the contribution of Mr. Priyam Mishra of Thapar University, Punjab, India in the development of the Android app which is used for data collection from the vehicles.

Open Access This article is distributed under the terms of the Creative Commons Attribution 4.0 International License (http:// creativecommons.org/licenses/by/4.0/), which permits unrestricted use, distribution, and reproduction in any medium, provided you give appropriate credit to the original author(s) and the source, provide a link to the Creative Commons license, and indicate if changes were made.

\section{References}

1. The New York Times, 7 March 1906 Page 3, - Newspapers.com Historical Newspapers from 1700s-2000s, accessed on 11 April 2015

2. Buchanan JA, Catudal AL, Greenshields B, Moyer R (1941) Standardizable equipment for evaluating road surface roughness. Highway Research Board Proceedings

3. Cundill M (1991) The MERLIN low-cost road roughness measuring machine. Transport and Road Research Laboratory

4. Morrow G (2006) Comparison of roughness measuring instruments. Master's Thesis, University of Auckland, Auckland, New Zealand

5. Sayers M, Gillespie T, Queiroz C (1986) The international road roughness experiment: a basis for establishing a standard scale for road roughness measurements. Transportation Research Record

6. Atkinson GM, Wald DJ (2007) "Did you feel it?" intensity data: a surprisingly good measure of earthquake ground motion. Seismol Res Lett 78:362-368

7. Reilly J, Dashti S, Ervasti M, Bray JD, Glaser SD, Bayen AM (2013) Mobile phones as seismologic sensors: automating data extraction for the iShake system. IEEE Trans Autom Sci Eng 10:242251
8. Herrera JC, Work DB, Herring R, Ban XJ, Jacobson Q, Bayen AM (2010) Evaluation of traffic data obtained via GPS-enabled mobile phones: the mobile century field experiment. Transp Res C Emerg Technol 18:568-583

9. Mohan P, Padmanabhan VN, Ramjee R (2008) Nericell: rich monitoring of road and traffic conditions using mobile smartphones. Proceedings of the 6th ACM Conference on Embedded Network Sensor Systems: ACM, pp 323-36

10. Eriksson J, Girod L, Hull B, Newton R, Madden S, Balakrishnan H (2008) The pothole patrol: using a mobile sensor network for road surface monitoring. Proceedings of the 6th International Conference on Mobile Systems, Applications, and Services: ACM, pp 29-39

11. Mednis A, Strazdins G, Zviedris R, Kanonirs G, Selavo L (2011) Real time pothole detection using android smartphones with accelerometers. Distributed Computing in Sensor Systems and Workshops (DCOSS), 2011 International Conference on: IEEE, pp 1-6

12. Ghose A, Biswas P, Bhaumik C, Sharma M, Pal A, Jha A (2012) Road condition monitoring and alert application: Using in-vehicle smartphone as internet-connected sensor. Pervasive Computing and Communications Workshops (PERCOM Workshops), 2012 I.E. International Conference on: IEEE, pp 489-91

13. de Frutos SH, Castro M (2014) Using smartphones as a very lowcost tool for road inventories. Transp Res C Emerg Technol 38: 136-145

14. Fazeen M, Gozick B, Dantu R, Bhukhiya M, González MC (2012) Safe driving using mobile phones. IEEE Trans Intell Transp Syst 13:1462-1468

15. Orhan F, Eren PE (2013) Road hazard detection and sharing with multimodal sensor analysis on smartphones. Next Generation Mobile Apps, Services and Technologies (NGMAST), 2013 Seventh International Conference on: IEEE, pp 56-61

16. Alessandroni G, Klopfenstein LC, Delpriori S, Dromedari M, Luchetti G, Paolini BD et al (2014) SmartRoadSense: collaborative road surface condition monitoring. UBICOMM 2014, The Eighth International Conference on Mobile Ubiquitous Computing, Systems, Services and Technologies, pp 210-5

17. Bhoraskar R, Vankadhara N, Raman B, Kulkarni P (2012) Wolverine: traffic and road condition estimation using smartphone sensors. Communication Systems and Networks (COMSNETS), 2012 Fourth International Conference on: IEEE, pp 1-6

18. Perttunen M, Mazhelis O, Cong F, Kauppila M, Leppänen T, Kantola J et al (2011) Distributed road surface condition monitoring using mobile phones. Ubiquitous Intelligence and Computing: Springe, pp 64-78

19. Forslöf L (2012) Roadroid-smartphone road quality monitoring. Proceedings of the 19th ITS World Congress

20. Tai Y-c, Chan C-w, Hsu JY-j (2010) Automatic road anomaly detection using smart mobile device. Conference on Technologies and Applications of Artificial Intelligence, Hsinchu, Taiwan

21. Douangphachanh V, Oneyama H (2013) A study on the use of smartphones for road roughness condition estimation. J East Asia Soc Transp Stud 10:1551-1564

22. Agostinacchio M, Ciampa D, Olita S (2014) The vibrations induced by surface irregularities in road pavements-a Matlab ${ }^{\circledR}$ approach. Eur Transp Res Rev 6:267-275

23. Jazar RN (2008) Vehicle dynamics. Theory and applications riverdale. Springer Science + Business Media, NY

24. Chopra AK (1995) Dynamics of structures. Prentice Hall, New Jersey 